\title{
L'aménagement des estuaires de la Vilaine et du Lay
}

\section{par G. Besnier}

Ingénieur Général du G.R.E.F.

\section{ef E. Leroy}

Ingénieur en Chef du Génie Rural

Chef du Service de l'Aménagement des Eaux de la Région des Pays de Loire

\section{Exposé général}

par G. BESNIER

Les estuaires des fleuves côtiers, se jetant dans l'Océan Atlantique, sont le siège d'une sédimentation qui pose de sérieux problèmes aux collectivités riveraines.

Par une assimilation hâtive à d'autres phénomènes, on a cru pendant longtemps que cette sédimentation avait pour origine l'érosion du cours supérieur du fleuve, avec floculation et précipitation des matériaux en suspension au contact des eaux douces avec l'eau salée.

En réalité, il convient de distinguer deux phases dans le phénomène.

Dans le passé, avant la dernière période glaciaire, l'érosion du cours supérieur des fleuves apportait à l'océan des quantités importantes de matériaux qui ont constitué au large, sur le plateau continental, un dépôt de sédiments fins.

Lorsque, à la fin de la dernière glaciation, le niveau des mers s'est abaissé de 30 à $40 \mathrm{~m}$, le lit des fleuves s'est creusé en conséquence et le bed Rock est partout entaillé jusqu'à la cote -40 .

Puis, le niveau des mers remontant, les estuaires ont été parcourus par des flots de marées importants. Ces flots et l'agitation en période de tempête remettent en suspension le stock de sédiments fins déposés au large. Entrant dans un estuaire disproportionné avec le débit actuel des rivières, le flot chargé de sédiments abandonne ceux-ci, comblant peu à peu les lits des fleuves côtiers.

C'est ainsi que se sont progressivement comblés les estuaires de la Vilaine, du Lay et de la Sèvre Niortaise. Cette explication du phénomène a donné lieu à de nombreuses controverses jusqu'aux études entreprises en 1960 à la demande de notre Service par le Laboratoire de Maisons-Alfort ainsi que par Mlle Durand et Kerfourn.

L'analyse des vases, le rapport kaolinite-Illite et l'analyse pollinique des dépôts a permis de montrer que, depuis 2500 ans, l'origine des dépôts était exclusivement marine. Même en période de crue, l'apport de sédiments venus de l'amont est insignifiant.

Je ne m'étendrai pas davantage sur ce point qui a déjà fait l'objet devant vous, le 22 mars 1968, d'une communication de $M$. Orgeron.

La conséquence de ce phénomène est l'envasement progressif des estuaires, la constitution de marais. Le fleuve maintient un lit qui a tendance à s'exhausser au-dessus des dépôts et, au cours des siècles, les hommes ont dû construire des digues pour stabiliser le lit et éviter ses divagations.

Enfin, depuis une cinquantaine d'années, on s'efforce de stopper le flot chargé de sédiments marins. Successivement ont été ainsi fermés l'estuaire de la Sèvre, à Marans, l'estuaire du Lay à l'Aiguillon et l'estuaire de la Vilaine à Arzal.

Nous nous proposons d'examiner les résultats obtenus en ce qui concerne la sédimentation et la dessalinisation sur deux de ces estuaires : Le Lay et la Vilaine.

\section{L'estuaire du Lay}

Le Lay est un petit fleuve côtier de Vendée dont le lit excède peu une centaine de kilomètres. Draînant $1600 \mathrm{~km}^{2}$ à son entrée dans le marais, le Lay a un régime 
semi-torrentiel pouvant débiter 4 à $600 \mathrm{~m}^{3} / \mathrm{s}$, avec un débit moyen annuel de quelques $\mathrm{m}^{3} / \mathrm{s}$ et un étiage quasi nul.

Le Lay se jette dans la baie de l'Aiguillon qui est le reste du golfe du Poitou, comblé en grande partie depuis le début de notre ère.

A la période Gallo-Romaine, les flots marins venaient battre les rives de la plaine calcaire et, sous Louis XIII, Luçon était un port très actif. En 2000 ans, le golfe du Poitou s'est réduit, en ce qui concerne la Vendée, de 66700 ha à la baie de l'Aiguillon de 5000 ha (fig. 1, carte de 1574; fig. 2, carte actuelle).

Le cours inférieur du Lay, régularisé, endigué par les travaux des moines puis des ingénieurs hollandais, comporte maintenant $15 \mathrm{~km}$ de trajet horizontal. Jusqu'en 1953 , les marées de vive eau remontaient entre les digues jusqu'au Gué de Mortevieille, $15 \mathrm{~km}$ en amont.

Le flot de marée, chargé de limon jusqu'à $15 \mathrm{~g} / \mathrm{l}$ de matières sèches en marée de vive eau, abandonne ainsi des dépôts impressionnants pendant la période d'étiage d'avril à novembre.

Lors des premières crues, une partie du dépôt remise en suspension repart vers l'aval. Mais, si la première crue est violente, le travail de dégagement ne peut s'effectuer à temps et l'amont en subit les conséquences.

En 1953, l'Association de la Vallée du Lay passait, avec l'aide du ministère de l'Agriculture, à l'exécution d'un programme comportant en particulier l'exécution du vannage du Braud, comportant six passes fermées par des vannes wagons.

L'effet obtenu a été immédiat et l'écoulement des crues, en aval de Mareuil, est assuré, depuis la mise en service de l'écluse du Braud, sans submersion des marais riverains.

Mais le phénomène de sédimentation se poursuit à l'aval du vannage, provoquant les réclamations des pêcheurs et conchyliculteurs du port de l'Aiguillon.

En effet, la baie de l'Aiguillon, avec ses 5000 ha, constitue un réservoir naturel entretenant la violence des courants de flots et de jusant. De ce fait, en marée de vive eau, le flot est sensiblement aussi chargé en vase que précédemment et l'envasement de la partie inférieure de l'estuaire se poursuit, d'où plaintes des usagers d'aval. Finalement, il a été décidé de laisser ouvertes les vannes du Braud de façon à bénéficier de l'effet de chasse produit par l'eau accumulée en amont du Braud. Toutefois, les vannes seront fermées dès que le coefficient de marée dépassera 75. En effet, le Laboratoire de Maisons-Alfort a montré qu'il existe une proportionnalité entre la puissance 4,64 de coefficient de marée et la quantité de vase transportée. En pratique, au-dessous du coefficient 80 , la turbidité est très faible.

Depuis l'application de ces mesures, l'effet de chasse produit en marée de morte eau et en marée moyenne suffit à dégager la partie aval du lit des vases déposées en vive eau.

\section{L'estuaire de la Vilaine}

Le problème de l'estuaire de la Vilaine est un problème similaire, mais à une autre échelle.

La Vilaine draine un bassin versant de $10000 \mathrm{~km}^{2}$ avec un cours de $225 \mathrm{~km}$. Son débit moyen est de $80 \mathrm{~m}^{3} / \mathrm{s}$,

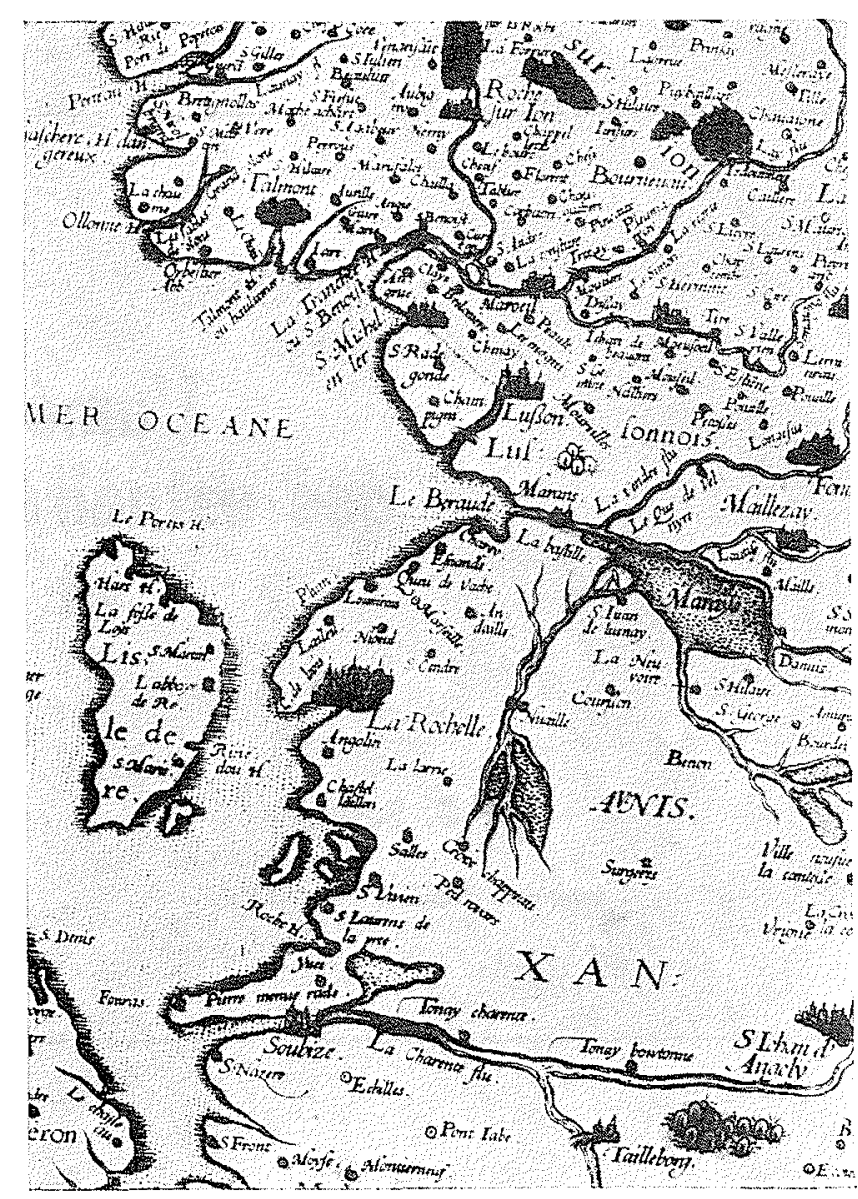

$1 /$

avec un étiage inférieur à $10 \mathrm{~m} \% / \mathrm{s}$, et des crues décennales dépassant $1200 \mathrm{~m}^{3} / \mathrm{s}$.

Son estuaire se prolongeait au-delà de Redon, mais, en 1934, un vannage établi à Redon limitait à $50 \mathrm{~km}$ la partie maritime du lit.

Ce lit, creusé dans le bed Rock jusqu'à des cotes -- 35 à - 40, a été remblayé jusqu'à la cote - -8 , les marais adjacents se trouvant à des cotes variant entre 2 et 2,50 N.G.F.

Le niveau des marées de vive eau pouvant atteindre 3,60 par fort vent d'ouest, l'ensemble des marais était recouvert d'eau salée plusieurs fois par mois.

Deux périodes pouvaient être distinguées dans l'année :

En période d'étiage, les vases remontaient vers l'amont et l'amplitude des dépôts d'été pouvait atteindre $2 \mathrm{~m}$ (fig. 3).

En période de crue, les vases érodées par le flot de crue redescendaient et allaient se déposer à l'entrée de l'estuaire. Il en résultait entre l'hiver et l'été un mouvement de bascule des sédiments et, finalement, la résultante était un envasement très lent qui peut être suivi depuis 1821 et qui n'excède guère $5 \mathrm{~mm}$ par an. Les analyses polliniques des sondages profonds par Mlle Durand et Kerfourn permettent d'évaluer à 2500 ans le délai de comblement des dix derniers mètres. On retrouve le taux de $5 \mathrm{~mm}$ par an (4 $\mathrm{mm}$ exactement).

Mais, si un équilibre s'établissait sur une année, les fluctuations du fond entraînaient un exhaussement du lit de deux mètres à la fin d'octobre, gênant l'évacuation des crues d'hiver.

Les premières crues, à l'entrée de l'hiver, avaient de la peine à s'écouler et il s'ensuivait des inondations des 


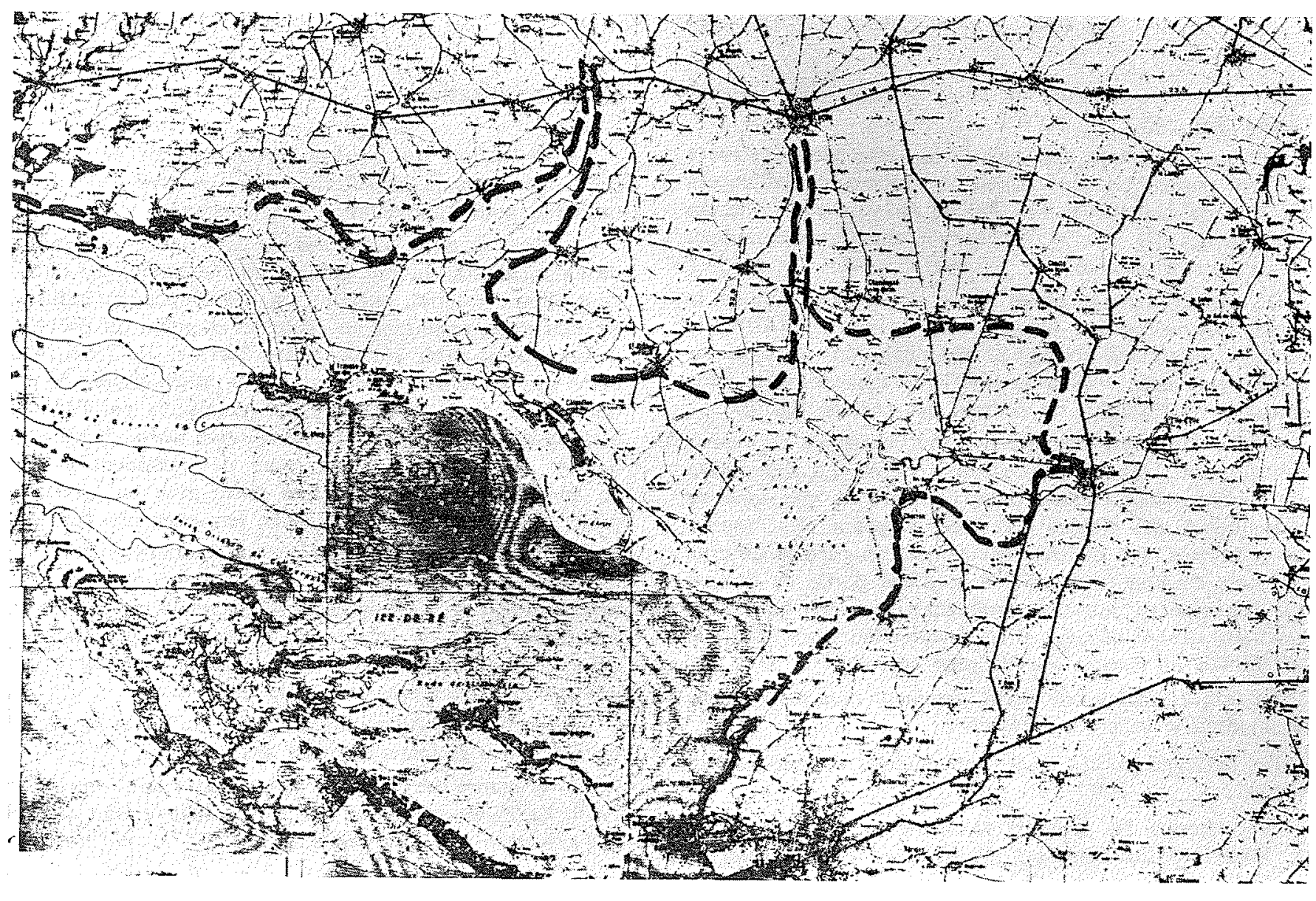

2/

(En trait tireté, le rivage de 1574.)

bas quartiers de Redon. En 1960, à la suite d'inondations plus importantes, un projet déjà ancien, puisque datant de 1936, fut adopté par l'Institution Interdépartemenale pour l'Aménagement de la Vilaine qui groupe les trois départements de Loire-Atlantique, Ille-et-Vilaine et Morbihan.

Ce projet consistait à fermer l'estuaire le plus à l'aval possible par un vannage (fig. 4). En amont de ce vannage, le volume du lit compris entre les marées basse et haute de vive eau est de $35000000 \mathrm{de} \mathrm{m}^{3}$. On pouvait donc espérer :

- assurer une meilleure évacuation des crues en bénéficiant entre marées basse et haute de cette capacité de stockage;

- limiter au vannage la remontée des flots chargés de limon;

- constituer en amont une réserve d'eau douce utilisable pour alimenter la région.

Les travaux adjugés à la fin de l'année 1965 ont duré cinq ans et la fermeture du lit a été assurée en novembre 1970.

Je me bornerai à examiner les deux points de la sédimentation et de la dessalinisation.

Je rappelle qu'il s'agit d'un estuaire de $50 \mathrm{~km}$ de longueur, avec des largeurs atteignant $500 \mathrm{~m}$.

L'importance des phénomènes de sédimentation a été mise en évidence durant les travaux.

Pour exécuter l'ensemble des vannages, une enceinte batardée de $45000 \mathrm{~m}^{2}$ fut réalisée sur la rive droite. L'exécution de ce batardeau nécessita une année et, avant la fermeture de l'enceinte dont le fond descendait de la rive

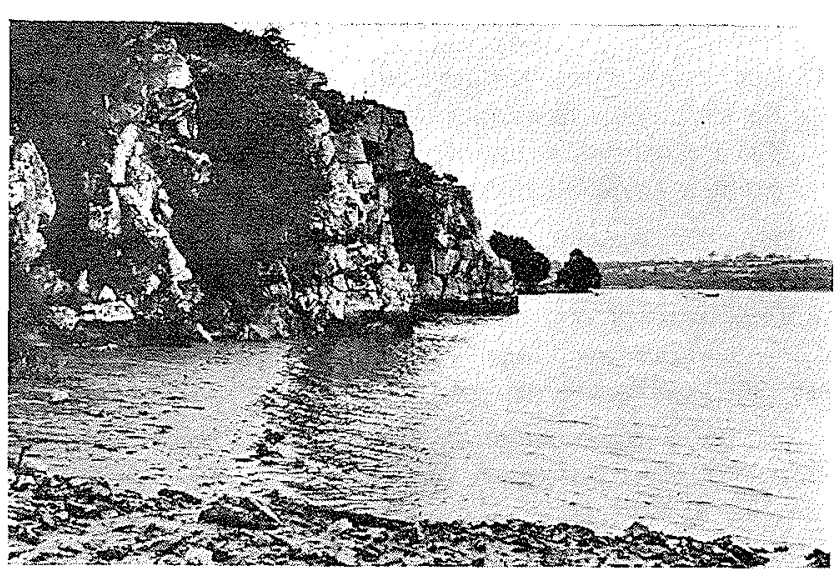

$3 /$

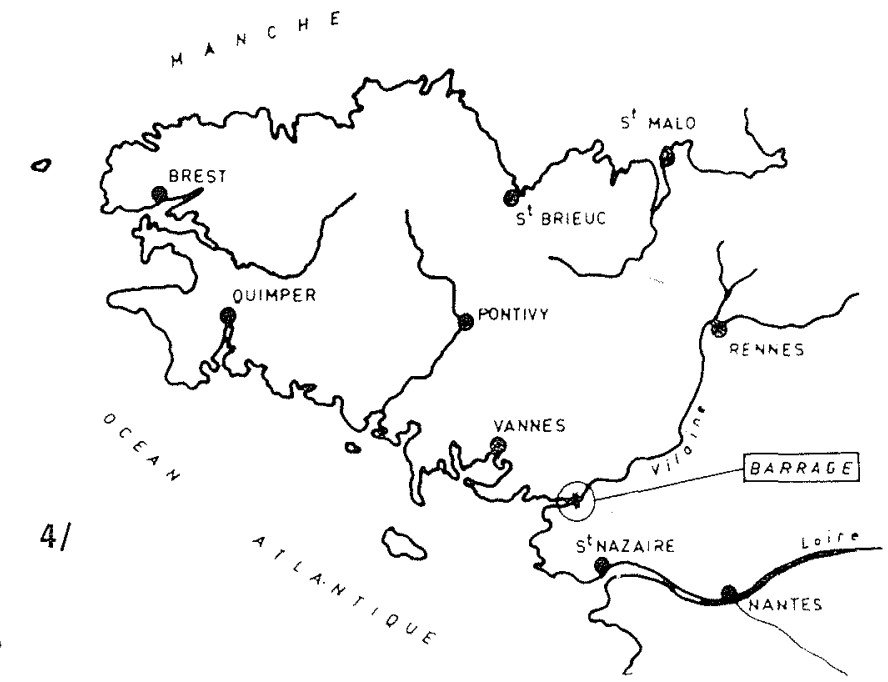


à la cote -10 , les eaux du fleuve y pénétraient naturellement (fig. 5).

Lorsque l'enceinte fut fermée et asséchée, il s'avéra que les parties les plus basses se trouvaient remblayées sur $4 \mathrm{~m}$ de hauteur et $115000 \mathrm{~m}^{3}$ de vase molle durent être évacués (fig. 6 et 7 ; la figure 8 montre le batardeau vu de la rive gauche).

De même, dans la phase finale de construction de la digue, lorsque celle-ci ayant atteint la cote 3 N.G.F., le courant se trouva coupé, la sédimentation remonta les fonds de la cote -8 à la cote -3 dans toute la zone amont et aval à l'abri du courant des pertuis (fig. 9).

Dès la fermeture du vannage, tout transport de vase a été supprimé, tant à l'amont qu'à l'aval. La diminution du volume d'eau entrant dans l'estuaire et, par voie de conséquence, des vitesses à l'entrée, supprime toute reprise des sédiments. Par contre, l'écoulement en période de crue des eaux d'amont a nettoyé le lit d'une partie de la vase qui l'encombrait jusqu'au droit du rivage maritime. Les bouchots se sont dégagés et les mytiliculteurs ont bénéficié de récoltes de moules sensiblement accrues.

En fait, le phénomène a été nettement plus marqué que ne l'indiquait le modèle réduit.

Les conclusions des essais sur modèles étaient les suivantes :

$1^{\circ}$ Les vitesses des courants de marée doivent être, après fermeture du vannage, insuffisantes pour assurer le transport de la vase dans un sens ou dans l'autre.

2" Seul le premier flot de marée de vive eau est suffisamment fort pour provoquer un transport de vase vers l'intérieur.

3" Les essais font ressortir un déplacement vers l'aval de Tréhiguier des zones de dépôts maxima, une absence de dépôts, voire une tendance à l'érosion immédiatement à l'aval du barrage.

Dans la pratique, toute sédimentation a totalement disparu d'Arzal jusqu'à la mer et les vases existantes ont été reprises par les courants de jusant. Ainsi, à l'aval immédiat de l'ouvrage, une cale, jadis recouverte de vase, est dégagée jusqu'à la cote -1 .

Le même phénomène de nettoyage a joué à Tréhiguier et jusque dans les zones de bouchots à l'aval de ce port.

Ainsi le résultat obtenu à Arzal est très positif alors que, sur l'estuaire du Lay, il est plutôt négatif. La raison en réside dans les rapports des volumes d'eau utilisés pour remettre la vase en suspension avant et après.

Sur le Lay, la vase est essentiellement reprise par le courant de flot de la baie de l'Aiguillon, soit 250 à 300000000 de $\mathrm{m}^{3}$ par cycle. Les quelque $1400000 \mathrm{~m}^{3}$ stockés ou non dans le lit du Lay ne modifient pas la grandeur du phénomène.

Sur la Vilaine, le volume d'eau transitant dans le fleuve représente 50 à $60000000 \mathrm{de} \mathrm{m}^{3}$. Le barrage d'Arzal réduit ce volume de moitié et les résultats sont là pour montrer que la réduction de vitesse en résultant, à l'entrée de l'estuaire, est suffisante pour stopper toute reprise des sédiments.

A l'aval d'Arzal, sur le parement du barrage, le phénomène de dévasement est concrétisé par une plage de sable sur laquelle les pêcheurs viennent échouer leur bateau pour les travaux de carénage et de peinture.

\section{La dessalinisation}

La réalisation du barrage d'Arzal devait entraîner, à brève échéance, la constitution d'une réserve d'eau douce utilisable pour l'alimentation des réseaux.

Or, tant au nord qu'au sud de la Vilaine, le problème de l'alimentation en eau potable ou industrielle est permanent. Toute solution entraîne un développement industriel et touristique remettant en cause les données du problème qu'on croyait avoir résolu. Notre Service, dès 1965, avait établi que les années au-delà de 1970 présenteraient de gros déficits dans ce domaine. C'est pourquoi, parallèlement à la construction du barrage, l'Institution demandait à notre Service la réalisation d'une usine de traitement à $3 \mathrm{~km}$ en amont de l'ouvrage. L'usine a été mise en service en juillet 1971, neuf mois après la fermeture de l'estuaire.

Indépendamment du problème de traitement des eaux, le problème des chlorures se posait avec toutes les incertitudes quant à leur disparition. Si une crue suffisait à renouveler l'eau de la réserve, il restait le problème des chlorures des vases et des terrains adjacents dont il était difficile d'apprécier le temps de disparition.

En pratique, la dessalinisation a été suffisante en surface en neuf mois et totale en deux ans et demi.

La fermeture des vannes a été effective le $1^{\text {cr }}$ novembre 1970. Le 19 novembre, en surface, la salinité était abaissée à $4 \mathrm{~g} / 1$ et en mai 1971 on relevait :

- $219 \mathrm{mmg} / \mathrm{l}$ à $1 \mathrm{~m}$ de la surface et jusqu'à $3 \mathrm{~m}$;

- $2847 \mathrm{mmg} / 1$ à $5 \mathrm{~m}$ de la surface.

En juin, à $5 \mathrm{~m}$, le taux s'abaissait à $650 \mathrm{mmg} / \mathrm{l}$. Mais le lit de la Vilaine n'est pas en pente régulière et le profil en long comporte une série de poches dont le fond peut s'abaisser à la cote -20 N.G.F.

Pendant toute l'année 1971, ces poches devaient rester remplies d'eau salée à $13 \mathrm{~g} / \mathrm{l}$ cependant que la station de traitement des eaux, située au droit d'une de ces poches, pompait en surface de l'eau dont la teneur en chlorure n'a jamais excédé pendant l'été 1971, $500 \mathrm{mmg} / \mathrm{l}$.

Les crues de l'hiver 1971 devaient abaisser le taux des chlorures dans les poches à $7 \mathrm{~g} / 1$.

Enfin, les crues de l'hiver 1972, pourtant peu importantes, ont abaissé le taux de chlorure à la valeur uniforme de $117 \mathrm{mmg}$, teneur très satisfaisante, le taux légal étant de $200 \mathrm{mmg} / 1 \mathrm{de} \mathrm{Cl}$, soit $320 \mathrm{mmg} \mathrm{NaCl}$.

\section{Conclusions}

La fermeture de l'estuaire a donc été bénéfique en ca qui concerne la Vilaine, tant pour l'amont que pour l'aval.

Indépendamment des problèmes de sédimentation et de régulation des crues, la solution apportée au problème de l'alimentation en eau potable est intéressante.

L'eau des estuaires est perdue définitivement et le débit permanent est, en général, bien supérieur aux besoins de la population et même de l'industrie.

Enfin, un estuaire est rarement plaisant à marée basse, avec les étendues de vases. La fermeture entraîne la création d'un plan d'eau valorisant l'arrière-pays par le développement du tourisme. Déjà, sur les 1500 ha du plan d'eau entre Arzal et Redon, les plaisanciers viennent admirer le site mis en valeur par les travaux de l'Institution. 


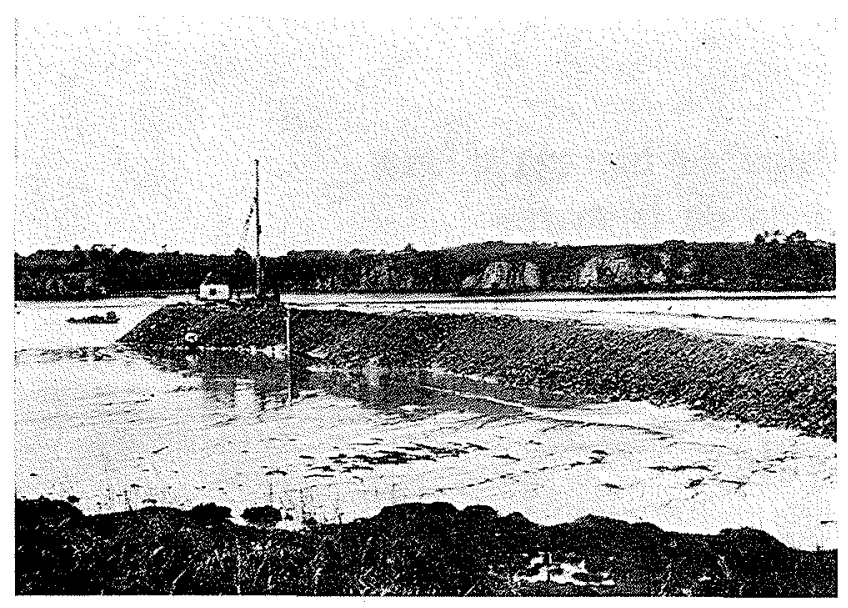

$5 /$

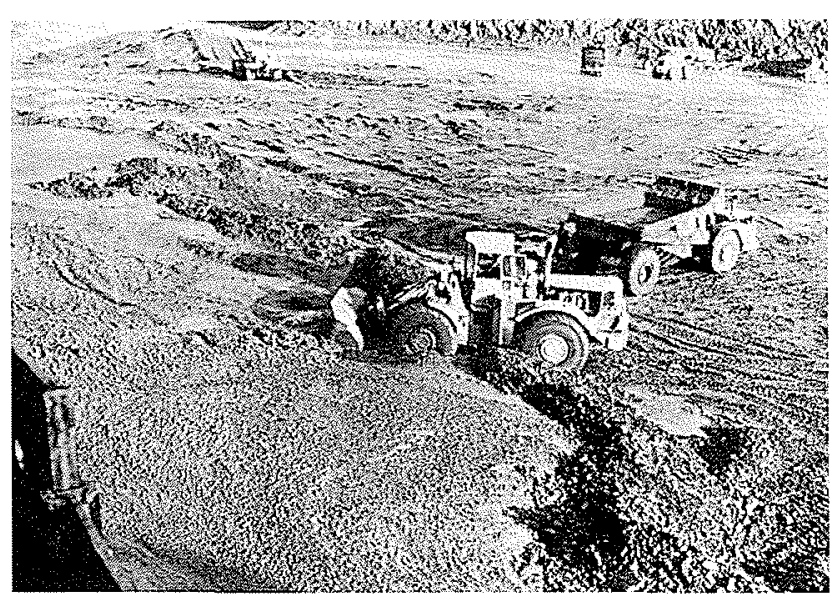

6/

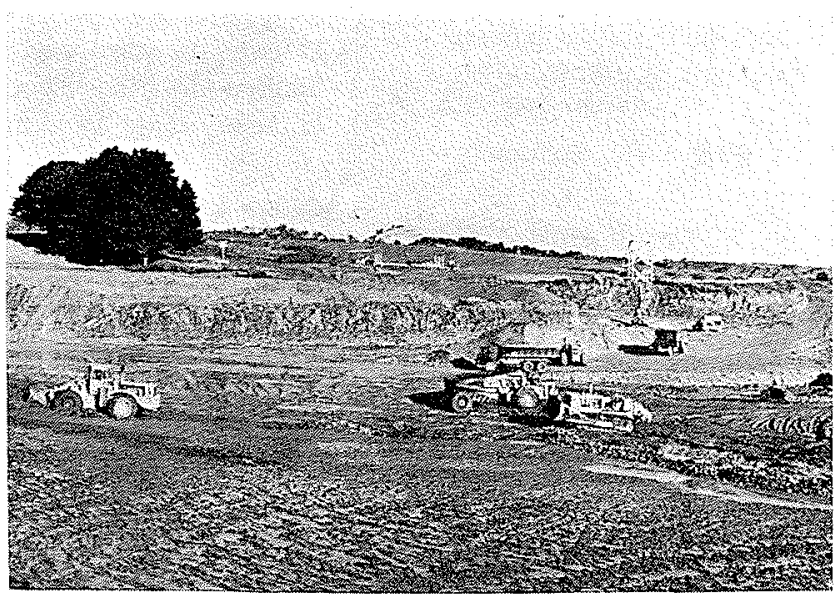

7/

4678

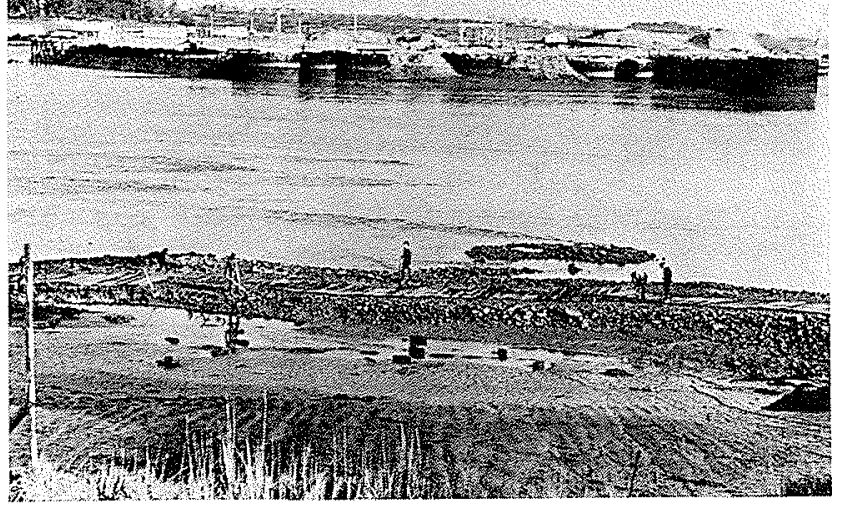

8/

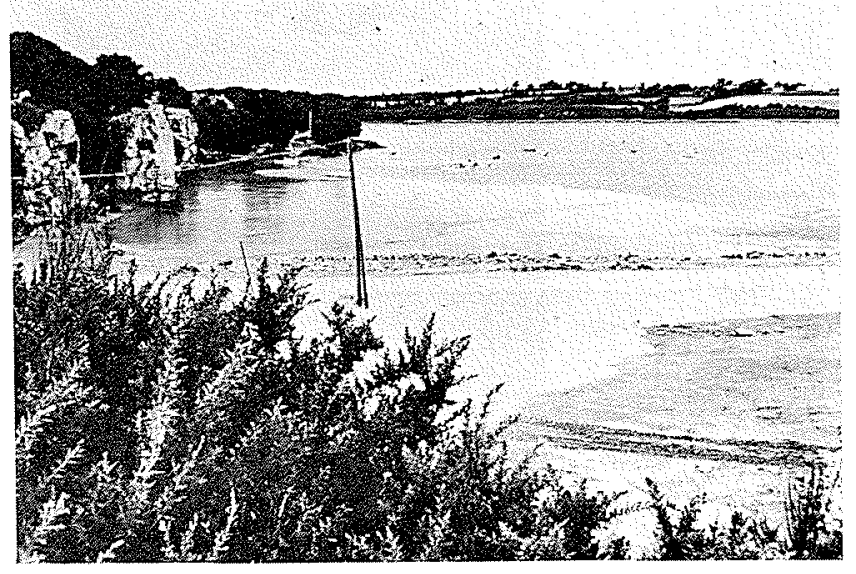

9/ 


\title{
Evacuation des crues de la Vilaine au barrage d'Arzal en fonction de l'importance de la marée
}

\author{
par E. LEROY
}

\section{Approche théorique du problème}

Depuis la mise en eau, fin 1970 , et en conséquence de la sécheresse hivernale qui sévit depuis quatre ans dans l'Ouest, le barrage d'Arzal n'a eu à évacuer aucune crue importante de la Vilaine. Cependant, de telles crues ne manqueront pas de survenir dans l'avenir et il est bon de se poser le problème de leur évacuation en fonction de l'importance de la marée.

L'évacuation des crues a déjà été étudiée par le Laboratoire d'Hydraulique de France en 1963-1964. Cependant, cette étude a été conduite pour des caractéristiques géométriques du barrage différentes des caractéristiques réelles d'exécution (radier à la cote - 4 alors qu'il est réellement à la cote -- 8 , largeur totale des cinq pertuis $100 \mathrm{~m}$, alors qu'elle est réellement de $90 \mathrm{~m}$ ).

La figure 10 montre le barrage tel qu'il a été réellement exécuté.

Par ailleurs, l'étude des pertes de charge à travers le barrage a été faite vannes grandes ouvertes, alors que la plupart des évacuations se font avec une ouverture limitée des vannes.

Enfin, le barrage ne se trouve pas, comme dans le modèle, sur un chenal de dérivation.

Nous utiliserons cependant, chaque fois que ce sera possible, les résultats du modèle pour caler les formules utilisées pour calculer l'écoulement.
Nous examinerons successivement :

a) les conditions d'écoulement à travers les pertuis;

b) les capacités de stockage dans la réserve;

c) le passage de différentes crues pour différentes marées.

\section{Etude de l'écoulement à travers un pertuis du barrage d'Arzal}

L'écoulement peut prendre des aspects différents selon les combinaisons des grandeurs qui le régissent et qui sont :

1. la cote du plan d'eau amont;

2. la cote du plan d'eau aval, qui varie avec la marée;

3. la position de la vanne.

Premier cas (fig. 11). - La vanne est suffisamment levée pour qu'elle ne touche pas le plan d'eau. Soit, par rapport au radier, $y_{1}$ le tirant d'eau amont et $y_{2}$ le tirant d'eau aval.

a) Si $y_{2} \leqslant 2 / 3 y_{1}$, le radier se comporte en seuil épais, il y a passage au régime critique, le débit ne dépend que de $y_{1}$.

Si la vitesse d'approche n'est pas négligeable, il y a lieu de remplacer $y_{1}$ par:

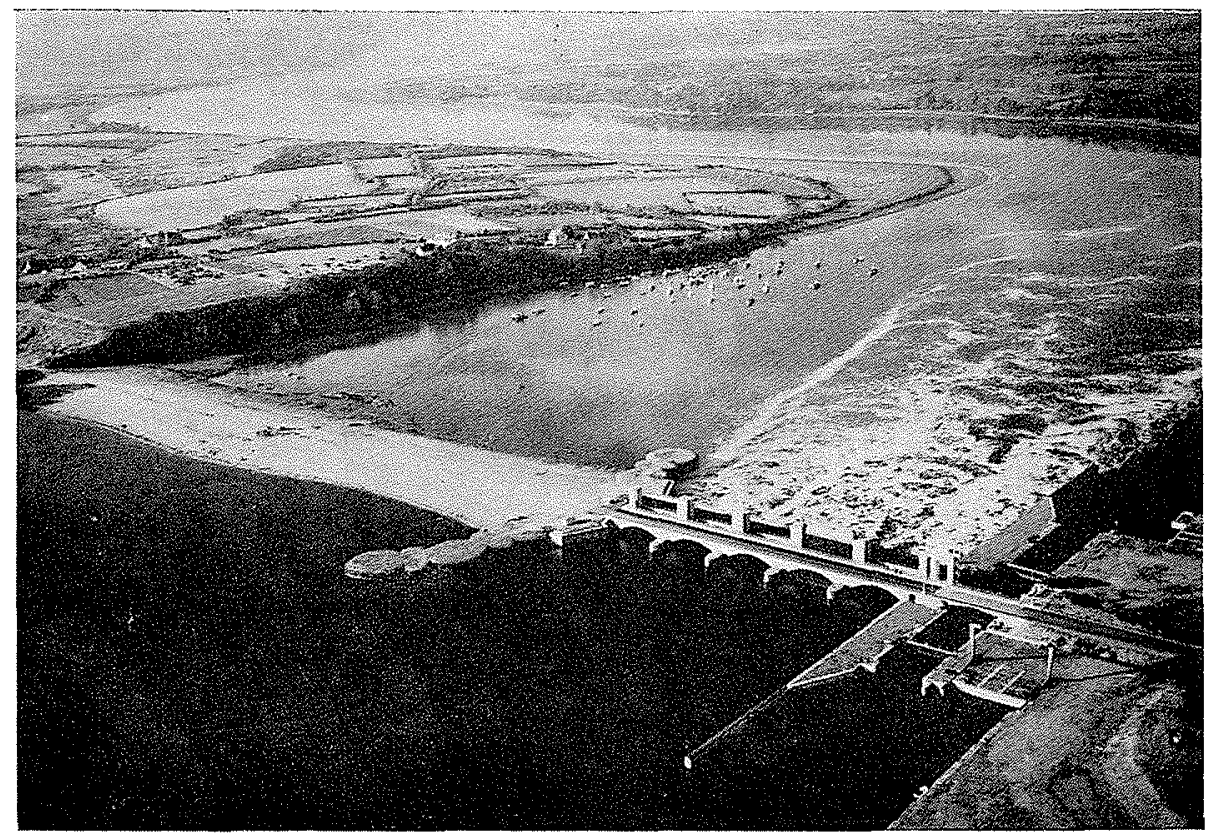




$$
H s_{1}=y_{1}+V_{1} \cdot 2 / 2 g
$$

b) Si $y_{2}>2 / 3 y_{1}$, l'écoulement est fluvial et le débit dépend de la différence $\left(y_{1}-y_{2}\right)$.

DeuxiÈme cas (fig. 12). - La vanne est levée d'une hauteur $h_{1}$, le bas de la vanne étant immergé.

a) La vanne est dénoyée par l'aval avec, pour certaines valeurs de $\gamma_{2}$, formation d'un ressaut hydraulique. Le débit ne dépend que de $y_{1}$

$$
q=m h_{1} \sqrt{2 g y_{1}} \text {. }
$$

(Ce cas se produira rarement, il nécessite à la fois un fort débit justifiant de lever la vanne d'une hauteur $h_{1}$ importante et une cote $y_{2}$ correspondant à une marée basse de fort coefficient).

b) La vanne est noyée par l'aval (hauteur h, du ressaut inférieure à $y_{2}$ ). Le débit par mètre de largeur de pertuis s'écrit : $q=n h_{1} \sqrt{2 g\left(y_{1}-y_{2}\right) \text {. }}$ S'agissant, dans le cas présent, de vannes verticales avec contraction sur un seul côté, nous prenons $n=0,7$.

Les conditions réelles d'exploitation du barrage se situeront presque toujours dans ce dernier cas.

On peut penser obtenir l'expression du volume évacué pendant un cycle de marée en intégrant l'expression mathématique du débit. It faudrait que l'ouverture des vannes reste constante pendant toute la marée, ce qui n'est pas le cas. Et, quand bien même la position des vannes et le niveau amont resteraient constants, le fait que $y_{2}$ niveau aval varie de façon sinusö̈dale avec la marée conduit à une intégrale elliptique.

Il n'y a pas d'expression mathématique simple du volume d'eau évacué au cours d'une marée.

Il faut donc rechercher une solution du problème par approximations successives.
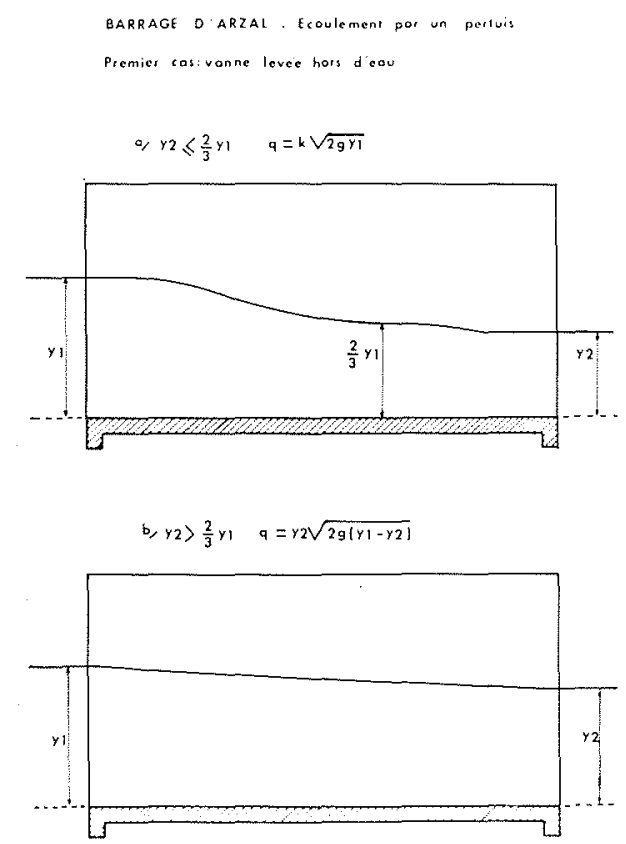

$11 /$
Appelons $Q(t)$ le débit d'apport dans la réserve à l'instant $t$. A ce même instant $t$, connaissant la position des vannes, les cotes $y_{1}$ et $y_{2}$, nous pouvons connaitre le débit évacué $q(t)$. Soit $V_{t}$ volume de la réserve à l'instant $t$.

Pendant un intervalle de temps $\Delta_{t}$ suffisamment court, nous pouvons négliger les variations de $Q(t)$ et de $q(t)$. Au temps $\left(t+\Delta_{t}\right)$ à la fin de l'intervalle de temps $\Delta_{l}$, le volume de la réserve sera :

$$
V\left(t+\Delta_{t}\right)=V_{t}+Q(t) \Delta_{t}-q(t) \Delta_{t}
$$

Si nous connaissons la fonction $Q(t)$ et la loi de variation de $V$ en fonction de $y_{1}$, nous pourrons calculer $y_{1}$ au temps $\left(t+\Delta_{t}\right)$ et, par conséquent, calculer $q\left(t+\Delta_{t}\right)$.

De proche en proche, il est possible de calculer l'évolution du niveau de la réserve connaissant :

a) $y_{1}$, donc $V$ au départ;

b) la variation $y_{22}(t)$ (marée);

c) la courbe $Q(t)$ des apports dans la réserve;

d) la courbe $V\left(y_{1}\right)$.

Le débit est supposé sans influence sensible sur le niveau $y_{2}$ à l'aval du barrage. Cette approximation est justifiée. En effet, à la cote marine 4, soit 1 N.G.F., la section du lit au barrage est de $2600 \mathrm{~m}^{2}$, un débit de $2000 \mathrm{~m}^{3} / \mathrm{s}$ y transiterait à une vitesse moyenne de l'ordre de $0,80 \mathrm{~m} / \mathrm{s}$. La hauteur correspondant à cette mise en vitesse est de l'ordre de $3 \mathrm{~cm}$. Le lit de la Vilaine va ensuite en s'élargissant à l'aval.

\section{Capacité de stockage dans la réserve}

La courbe $V\left(y_{1}\right)$ est connue par des relevés topographiques malheureusement assez anciens. Ces relevés permettent d'établir la courbe $V\left(y_{1}\right)$ à débit nul (plan d'eau horizontal).

Le modèle réduit, exécuté par le Laboratoire d'Hydraulique, a permis de connaitre les pertes de charge pour

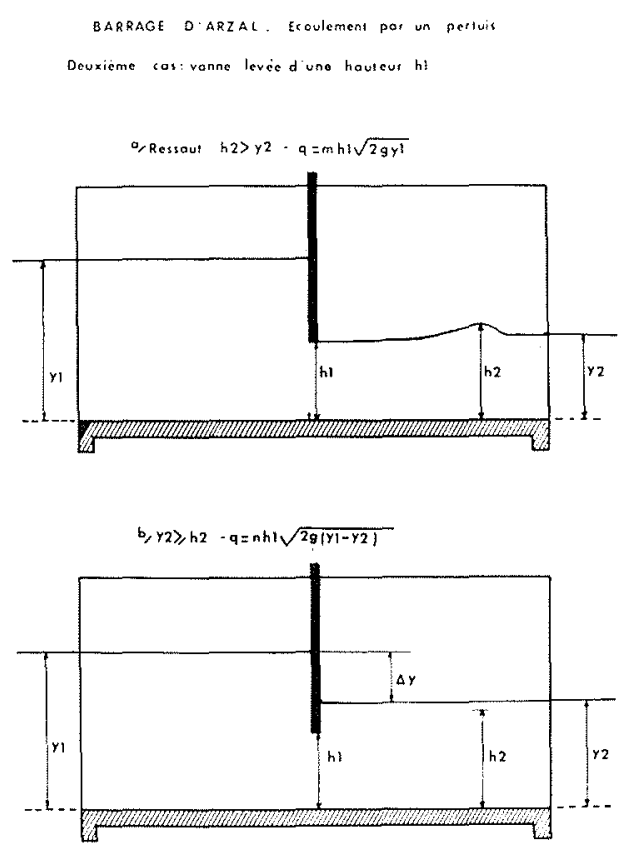

$12 /$ 
différents débits entre le barrage d'Arzal et le lieu-dit de Bellion situé $32 \mathrm{~km}$ à l'amont.

Ces pertes de charge sont fortes dans le cours amont plus étroit.

Ces pertes de charge sont également fortes dans la partie située immédiatement à l'amont du barrage, du fait sans doute de la présence du chenal mis en place sur ce modèle.

Dans la situation réelle actuelle, le lit va en s'élargissant constamment et les pertes de charge au kilomètre doivent diminuer constamment de l'amont vers l'aval.

Le calcul de proche en proche, profil par profil, permet d'établir la courbe de remous entre ces deux points (fig. 13). Nous avons utilisé la formule de Manning Strickler; pour obtenir par le calcul la perte de charge déterminée par le modèle réduit, il faut donner au coefficient $K_{\text {, la }}$ valeur 50. Le réseau des courbes de remous a été déterminé pour trois débits $\left(1560,1050,700 \mathrm{~m}^{3} / \mathrm{s}\right)$. Un seul est présenté en figure 13. Pour chacun de ces trois débits, en utilisant le réseau des courbes de remous, il a été possible de déterminer les courbes $V\left(y_{1}\right)$ (fig. 14).

La pente de ces courbes diminue légèrement lorsque le débit augmente, ce qui diminue d'autant la capacité de stockage d'une tranche d'eau donnée, même si le stock global est supérieur pour une même cote.

\section{Passage de différentes crues pour deux types de marée}

Nous avons, pour différentes crues et deux types de marée, calculé par approximations successives l'évolution du tirant d'eau $y_{1}$.

Le pas de temps $\Delta_{t}$ choisi pour les calculs a été de un quart d'heure, sauf pendant les périodes de manœuvre des vannes où il a été de trois minutes.

Compte tenu de l'importance du bassin versant de la Vilaine à Arzal $\left(10000 \mathrm{~km}^{2}\right)$ les crues ne surviennent pas brusquement. Le débit de crue a été supposé constant pendant la dizaine d'heures couverte par chaque calcul de variation du plan d'eau. Les crues ont été estimées en débit moyen journalier.

La courbe de marée a été construite à partir des heures et niveaux de haute et basse mer données par les annuaires, en utilisant la règle des douzièmes.

\begin{tabular}{|c|c|}
\hline $\begin{array}{c}\text { Fraction du TEMPS } \\
\text { ENTRE HAUTE ET BASSE MER ET } \\
\text { ENTRE BASSE ET HAUTE MER }\end{array}$ & Fraction DE L'AMpitudude \\
& \\
\hline & \\
\hline $1 / 6$ & $1 / 12$ \\
$2 / 6$ & $3 / 12$ \\
$3 / 6$ & $6 / 12$ \\
$4 / 6$ & $9 / 12$ \\
$5 / 6$ & $11 / 12$ \\
$6 / 6$ & $12 / 12$ \\
\hline
\end{tabular}

La vitesse de translation verticale des vannes est de $165 \mathrm{~mm}$ par minute. Pour la commodité des calculs, nous avons arrondi à $50 \mathrm{~cm}$ en trois minutes (erreur $1 \%$ ).

Notons préalablement qu'à marée basse, cote marine + 2 et pour une dénivelée de $1 \mathrm{~m}$, les vannes étant entièrement levées, la section de passage est de :

$$
5 \times 18 \times 7=630 \mathrm{~m}^{2}
$$

La mise en vitesse correspondant au $\Delta_{y}==1 \mathrm{~m}$ est de $4,429 \mathrm{~m} / \mathrm{s}$.

Si nous pratiquons un abattement de $10 \%$ pour tenir compte des frottements, le débit évacué serait de :

$$
4 \times 630=2520 \mathrm{~m}^{3} / \mathrm{s}
$$

Il n'y aura donc pas de problème pour vidanger la réserve à marée basse. Si la cote amont est de +1 , la dénivelée serait de $2 \mathrm{~m}$, la vitesse de $6264 \mathrm{~m} / \mathrm{s}$, et le débit de $6264 \times 630 \times 0,9=3550 \mathrm{~m}^{3} / \mathrm{s}$.

Nous examinons en premier lieu (fig. 15) l'évacuation d'une crue importante de $1550 \mathrm{~m}^{3} / \mathrm{s}$ au cours d'une marée de morte eau (coefficient 28); nous avons choisi dans un annuaire de marées celle du 27 février 1973.

Il n'y a aucune difficulté à évacuer le débit à marée haute sous une charge de $50 \mathrm{~cm}$. Le plan d'eau amont peut être maintenu à la cote 1,00 N.G.F. par manœuvre progressive des vannes. La constance de ce niveau ne sera pas absolue, elle dépendra de l'habileté dont fera preuve l'éclusier dans la manœuvre des vannes.

Considérons maintenant une marée de vive eau (coefficient 115). Nous avons choisi dans l'annuaire la marée du 17 mars 1972.

La figure 16 simule la manœuvre habituelle en cas de petite crue $\left(400 \mathrm{~m}^{3} / \mathrm{s}\right)$ :

Lorsque la marée monte et que son niveau approche du niveau amont, on ferme les vannes, à raison de $0,50 \mathrm{~m}$ par trois minutes, de telle sorte que la fermeture complète intervienne avant que le $\Delta_{y}$ tombe au-dessous de 0,25 à $0,30 \mathrm{~m}$, ceci afin d'éviter toute entrée d'eau salée dans la réserve.

En effet à $10^{\circ}$, l'eau salée à $30 \%$ a pour poids spécifique $1,023 \mathrm{~kg}$ par litre, il faut donc une colonne d'eau douce de $10,23 \mathrm{~m}$ pour équilibrer une colonne d'eau salée de $10 \mathrm{~m}$.

Pendant la haute mer, le débit de crue est stocké, l'ouverture des vannes commence dès que, à marée descendante, le niveau amont dépasse le niveau aval d'une trentaine de centimètres. Le volume mis en stock sera vidangé à marée basse, etc.

Pour une crue plus importante : $700 \mathrm{~m}^{3} / \mathrm{s}$ (crue théoriquement annuelle sur la Vilaine), la figure 17 montre que, pour la même marée et une cote de départ un peu plus basse, la durée de fermeture augmente, Je volume à stocker augmente et que le temps de manœuvre des vannes n'est pas négligeable, le niveau continuant à monter pendant la manceuvre d'ouverture des vannes.

Le niveau au Bellion (32 km à l'amont) est obtenu en ajoutant au niveau au barrage la perte de charge lue sur le réseau des courbes de remous. Ce niveau amont n'est qu'une approximation; la courbe ainsi construite sera décalée dans le temps et vraisemblablement déformée, car le temps de parcours des ondes entre les deux points dépend du tirant d'eau qui est lui-même variable.

Le modèle réduit a montré que le temps de propagation de l'onde de fermeture jusqu'au pont de Cran était de $1 \mathrm{~h}$ à $1 \mathrm{~h} 30$, le niveaul étant relativement bas, l'onde d'abaissement à l'ouverture devrait se propager plus vite, le niveau moyen étant plus élevé à l'ouverture qu'à la fermeture.

Il n'a pas été tenu compte de ces ondes dans le figuré 


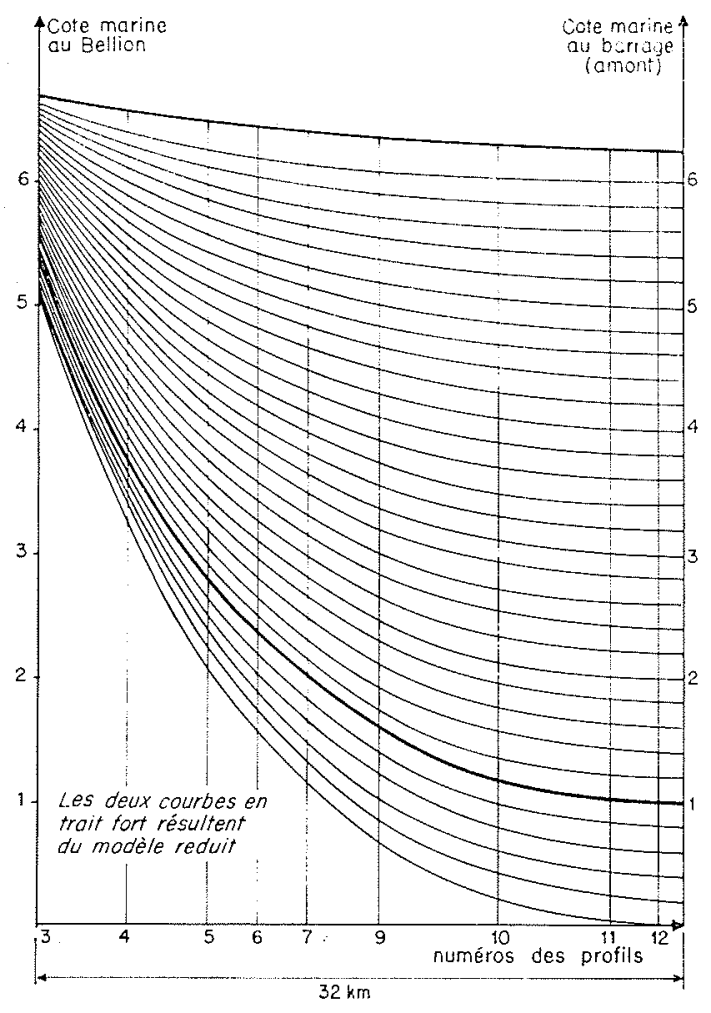

13/

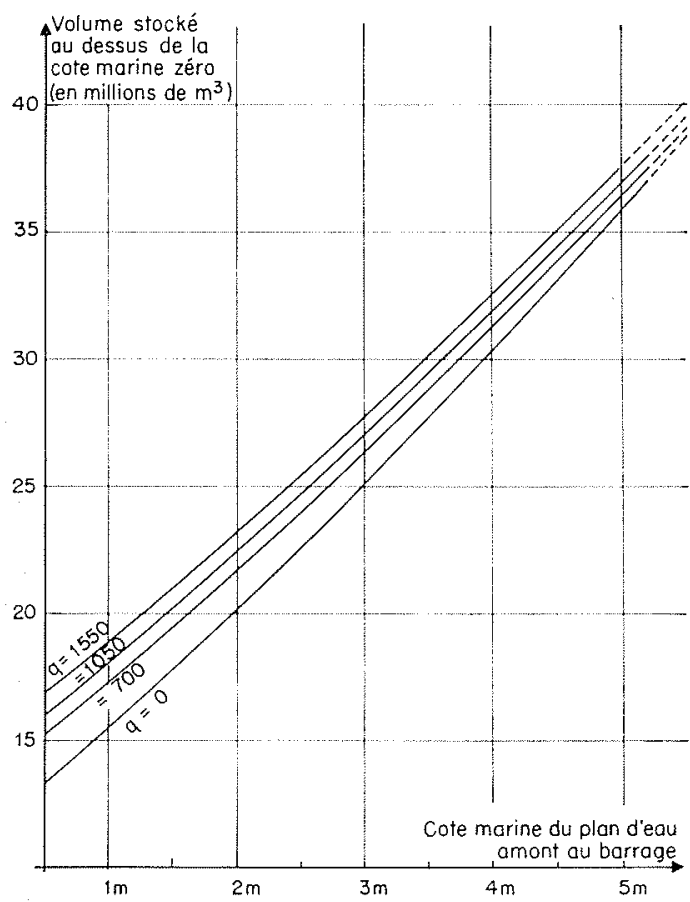

$14 /$

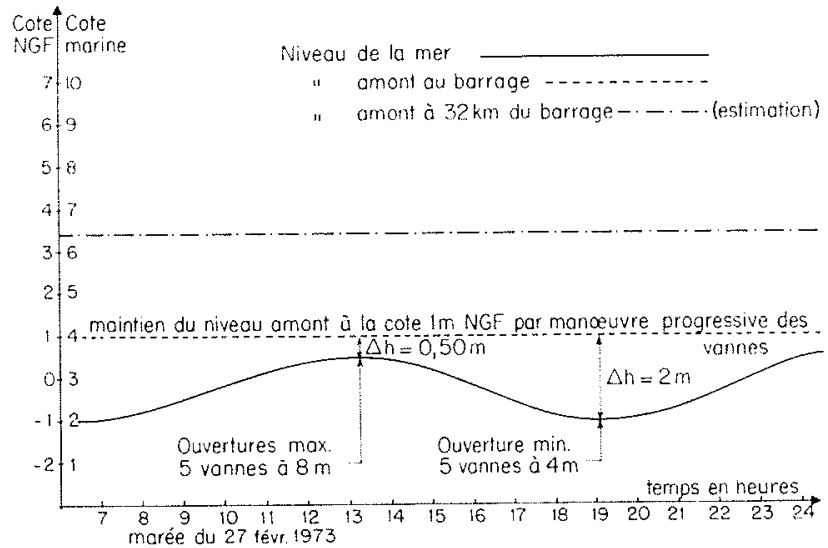

15/

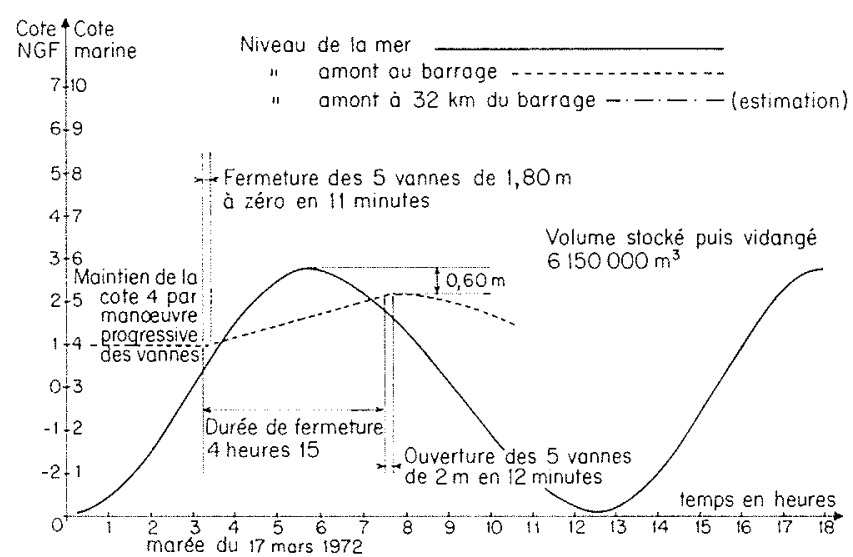

$16 /$

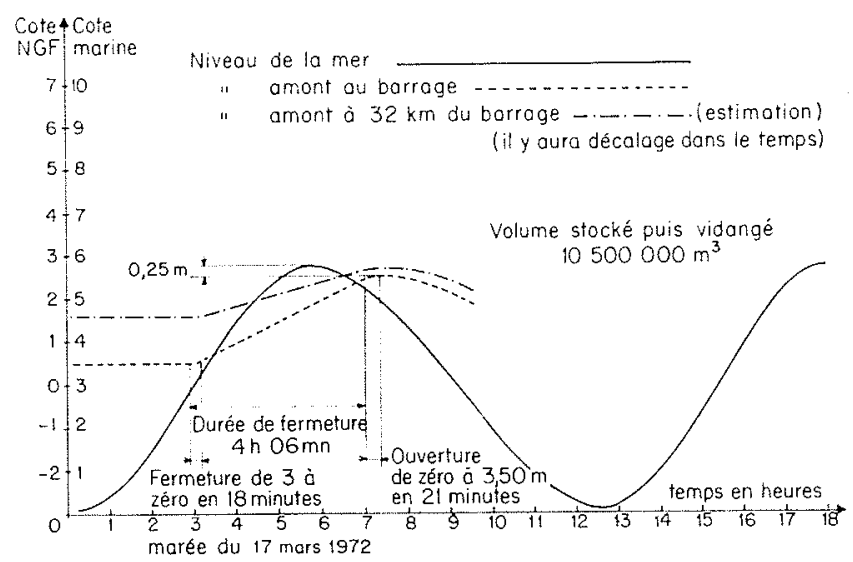

$17 /$ 


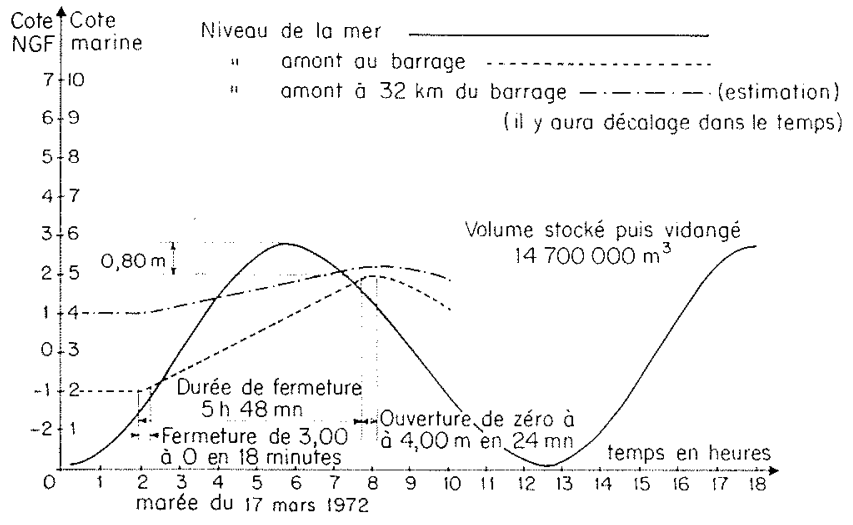

$18 /$

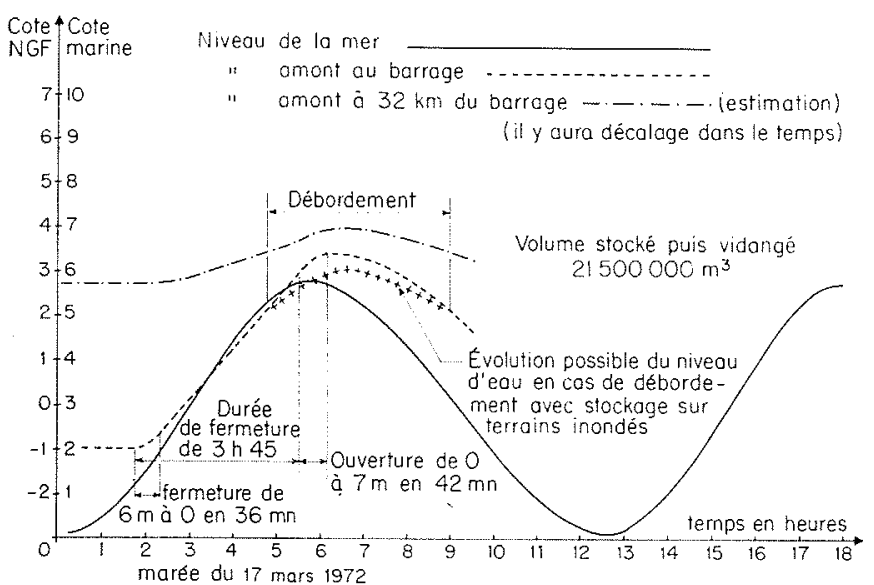

$19 /$

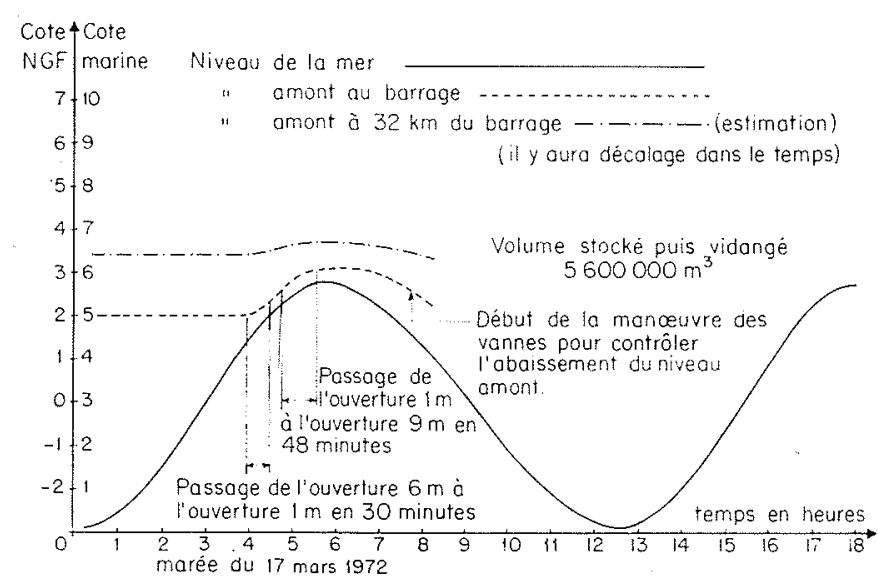

$20 /$ des niveaux amont en fonction du temps. Notons que l'onde de fermeture peut être amortie par déversement par-dessus les clapets qui surmontent les vannes.

Pour gagner plus que $0,25 \mathrm{~m}$ sur la cote de la haute mer, au même débit, il vient naturellement à l'esprit d'abaisser préalablement le niveau amont pour obtenir un "creux» appréciable et y stocker la crue (fig. 18).

Par rapport au cas de la figure 17, un abaissement supplémentaire préalable de $1,50 \mathrm{~m}$ du niveau amont permet de gagner $0,55 \mathrm{~m}$ seulement sur la cote maximum atteinte. La durée de fermeture est portée à $5 \mathrm{~h} 48 \mathrm{mn}$. Nous pressentons que cette voie ne sera pas toujours praticable pour des débits supérieurs.

Aul débit de $1550 \mathrm{~m} 3 / \mathrm{s}$ (fig. 19), compris entre la crue décennale et la crue centenaire, et en partant d'un niveau amont très bas, on s'aperçoit que le plan d'eau amont, vannes fermées, monte sous l'effet de la crue aussi vite que la marée et que l'ouverture doit intervenir un quart d'heure avant la marée haute. L'ouverture importante nécessaire pour évacuer $1550 \mathrm{~m}^{3} / \mathrm{s}$ sous une charge faible au départ, n'est obtenue qu'en $42 \mathrm{mn}$. Pendant ce temps, le niveau amont atteint la cote 6,40. Au-delà de la cote 5,00 et pour ce débit, les débordements doivent se produire à l'amont. Le plan d'eau n'atteindrait la cote 6,40 que pour une rivière endiguée [extrapolation de la courbe $Q\left(y_{1}\right)$ figure 13].

Dans la réalité, le débordement doit permettre de stocker le même volume sous une cote plus faible et la courbe réelle d'évolution du plan d'eau amont pourrait être la courbe $t+t+t+t+$ (fig. 19).

Cette politique de création et de remplissage d'un creux revient finalement, pour un fort débit, à reproduire la marée avec un léger décalage horaire à l'amont immédiat du barrage. De telles variations seraient désagréables pour l'usine de traitement d'eau potable do Drezet et le port de plaisance de la Roche-Bernard.

A $32 \mathrm{~km}$ à l'amont ces variations seraient très amorties.

Une autre politique paraît possible (fig. 20). Maintenir à marée basse, et le plus longtemps possible, un niveau amont proche de la cote de débordement. Fermer progressivement les vannes pour conserver toujours $30 \mathrm{~cm}$ de dénivelée entre l'amont et l'aval, sans aller jusqu'à la fermeture totale, puis, dès que la marée monte moins vite que le plan d'eau amont, ouvrir pour augmenter au maximum le débit d'évacuation. Une telle politique permet de gagner $25 \mathrm{~cm}$ environ sur la cote maxinum du plan d'eau amont, mais elle comporte des risques de mauvaise appréciation de la situation et d'introduction d'eau salée.

La politique de stockage et vidange peut présenter certains avantages et permettre, par exemple, le drainage alternatif de terrains endigués drainés par des ouvrages à clapet.

Compte tenu des imperfections et des approximations de la méthode utilisée, cet exposé ne prétend pas apporter la solution exacte de l'évacuation des crues de la Vilaine par marée de vive eau.

Cependant, certains problèmes ont été mis en lumière qui se présenteront un jour ou l'autre:

Comme le modèle réduit l'avait montré, la présence du barrage ne permettra pas d'éviter les inondations à l'amont en cas de fortes crues. Ces inondations sont liées à la capacité de transit du lit. Elle permettra, par contre, d'éviter ces inondations en cas de forte marée et de crue ordinaires (durée de retour inférieure à 10 ans). 
La situation la plus dangereuse serait créée par la conjonction d'une forte crue et d'une marée de vive eau. Cette situation peut très bien se produire en mars, à l'occasion des grandes marées d'équinoxe.

Les périodes de crues de la Vilaine à la station de jaugeage de Mâlon ont été, d'après les mesures de la Circonscription Electrique :

En 1964 :

- du 20 au 28 février;

- du 16 au 27 mars.

\section{En 1965 :}

- le 31 janvier et le $1^{\text {er }}$ février;

- du 19 al 24 février;

- du 22 al 25 mars;

- du 30 novembre au 3 décembre;

- du 26 au 31 décembre.

\section{En 1967 :}

- du $1^{\mathrm{er}}$ au 4 janvier;

- le 20 janvier;

- du 26 au 28 janvier;

- du 19 au 25 février;

- du $1^{\text {er }}$ au 4 mars;

- les 5 et 6 novembre;

- les 25 et 26 décembre.

\section{En 1968 :}

- du 3 au 14 janvier;

- du 7 au 9 février;

- du 22 au 24 février;
En 1969 :

- les 14 et 15 janvier;

- du 20 au 25 février;

- du 18 au 21 mars.

Trois ans sur cinq, des crues se sont produites vers le 20 mars, date des grandes marées. Le risque de conjonction forte crue-grande marée existe donc, mais il paraît difficile d'en chiffrer la probabilité.

Comme de bonnes mesures valent toujours mieux que des calculs, nous avons proposé d'équiper le barrage d'Arzal d'un enregistreur à deux niveaux. Un tel enregistreur sera prochainement installé dans la cabine de commande des vannes. Cet enregistreur tracera sur le même diagramme les deux courbes du niveau amont et du niveru aval, et permettra aux responsables, en fonction des résultats encore visibles de la marée précédente et de l'évolution en cours des niveaux d'eau, d'être mieux éclairés pour choisir une politique d'évacuation de la crue.

\section{Discussion}

Président : M. J. Chapon

M. le Président remercie vivement M. LEROY qui a présenté très clairement deux problèmes d'estuaires assez différents de ceux traités au cours de cette séance : problème de sédimentation d'un estuaire compètement et artificiellement barré: problème de l'évacuation des crues dans un estuaire soumis à la marée et fermé par un barrage.

Il ouvre ensuite la discussion.

M. Lamouroux souhaite avoir quelques informations sur le tassement actuel du barrage d'Arzal.

Ce tassement, répond $M$. Leroy, suit approximativement une loi logarithmique, c'est-à-dire que, si ce tassement a été de $x$ dans les premiers dix ans, il atteindra $2 x$ en 100 ans, et $3 x$ en 1000 ans. Dès maintenant, ce tassement est pratiquement stabilisé. D'ailleurs, lentreprise qui a construit l'ouvrage doit, par contrat, maintenir constante la cote du niveau de sa crête pendant un certain nombre d'années.

Est-ce que des études écologiques ont été faites avant - et aussi après - la construction du barrage en vue d'évaluer les conséquences de celui-ci sur la faune et la flore des fonds? demande M. Toussaint.

Je sais, répond $M$. le Président, que des études très sérieuses ont été faites pour essayer, malgré de très nombreuses difficultés dues au fait qu'on ne pouvait pas faire l'historique et que la situation, par conséquent, n'évoluait pas (au moins statistiquement) - d'évaluer l'évolution des fonds et les conséquenses que cette évolution pouvait avoir sur le plan de la salinité et de la production dans les bouchots.

Par contre, on ne peut pas dire qu'on ait fait des études écologiques pour connaître avec précision les évolutions prévisibles de la flore et la faune dans les zones avoisinantes. Mais, à l'époque, on ne parlait pas encore d'écologie.

M. Crouzet (Agence de Bassin Loire-Bretagne) apporte les informations ci-après :

Sur le plan écologique, la Faculté des Sciences de Brest fait des études sur les problèmes posés par les modifications du milieu dues aux barrages d'estuaires. Cela va beaucoup plus loin que le simple remplacement de l'eau salée par de l'eau douce; cela influence beaucoup les peuplements d'animaux migrateurs, principalement les crevettes. Si l'évolution est bénéfique sur le plan des bouchots à moules, qui vont être dévasés, et aussi sur le plan de la pollution, par contre on voit disparaitre les crevettes et certains poissons du type «mulet» qui vivent partie en rivière, partie en mer, et qui aiment les eaux dessalées et les gradients de salinité.

M. Leroy précise : les vannes du barrage d'Arzal sont surmontées de clapets qui servent à l'évacuation des petits débits, des corps flottants et, éventuellement, des pollutions du plan d'eau par hydrocarbures. Quand la marée monte, ces clapets se ferment automatiquement pour une différence de niveau d'environ $0,30 \mathrm{~m}$ entre l'amont et l'aval. Or, juste avant la fermeture, on voit des mulets sauter de la mer vers l'amont. Le barrage n'a donc pas interrompu leur migration; d'ailleurs les pêcheurs le savent bien.

\section{Valadou pose ensuite les deux question ci-après :}

$1^{\circ}$ Est-ce que les divers estuaires des bassins côtiers de la Vendée et de la Bretagne sont menacés d'envasement marin, comme cela se produisait pour la Vilaine, le Lay et la Sèvre niortaise?

$2^{\circ}$ Peut-on imaginer une autre solution que le barrage en estuaire; par exemple, des chasses violentes provenant des barrages-réservoirs amont? Sinon, à terme, tous les estuaires côtiers ne risquent-ils pas d'être fermés?

A la première question, M. le Président répond :

Cette tendance au comblement naturel des estuaires est curieusement appelée la régularisation des côtes. C'est une manifestation hydraulique et sédimentologique générale car, là comme ailleurs, " la nature a horreur du vide ».

Des essais théoriques d'estuaires ont été exécutés au Laboratoire Central d'Hydraulique à Maisons-Alfort. On avait choisi un estuaire de section à décroissance exponentielle de l'aval vers l'amont, à l'amont duquel était injecté un petit débit fluvial. Autant le phénomène de sédimentation progressive de l'estuaire était très rapide quand il n'y avait pas de débit fluvial, autant il suffisait d'un débit fluvial relativement faible pour réduire considérabement ce phénomène de «régularisation 》.

Par conséquent, je crois qu'il y a une «régularisation» très 
lente qui s'effectue dans toutes les embouchures de fleuves côtiers dont vous avez parlé. Mais, comme un débit d'amont intervient, le phénomène n'est pas aussi rapide que si l'on avait vraiment une rivière sans aucun apport liquide venant de l'amont.

La deuxième question de $M$. Valadou fait l'objet d'un bref débat que M. le Président résume comme suit :

Procédé classique, étudié encore récemment pour l'estuaire de la Canche, le bassin de chasse ne peut être utilisé dans tous les cas ct présente certains aléas. Tout d'abord, il convient que les débits de chasse soient au moins du même ordre que les débits engendrés dans l'estuaire par la marée; or, ces derniers sont souvent fort importants; ainsi, le débit fluvial de la Seine est quasi-négligeable vis-à-vis du « débit de marée ». Pourtant, on a constaté qu'une crue de quelques centaines de $\mathrm{m}^{3} / \mathrm{s}$, transitant au droit d'Honfleur, avait toujours un effet bénéfique.

Pour pouvoir compter sur un réservoir de chasse, il faut éviter son envasement et cela peut conduire à des travaux d'entretien fort coûteux.

Personne ne demandant la parole, M. le Président clôt la discussion.

11 tire ensuite, comme suit, les conclusions de la séance :

Nos conférenciers vous ont entretenus successivement de l'estuaire de la Seine, de l'estuaire de la Loire, de la Gironde puis des estuaires de petites rivières côtières comme la Vilaine et le Lay. Ce qui caractérise tous ces estuaires, c'est l'extrême diversité des phénomènes dont ils sont l'objet.

Dans un cas, se posent des problèmes de sable fin mis en mouvement par des courants relativement importants : c'est la Seine. En Loire, on parle de sables grossiers on de «bouchon vaseux». Dans la Gironde, on met l'accent sur les sables, mais surtout sur la vase et une marée qui va s'amplifiant en allant vers l'amont. Dans la Vilaine, on s'intéresse essentiellement à la vase.

Il y a donc une diversité de situations qui se retrouve dans les méthodes d'étude et d'aménagement.

Les progrès des modèles mathématiques et des modèles physiques permettent aujourd'hui, dans ce domaine, de résoudre - non pas à coup sûr, mais avec une très bonne probabilité de réussite - les problèmes les plus difficiles si l'on a suffisamment de données ì introduire dans lesdits modèles.

A cet égard, il faut être conscient de la difficulté des études concernant ces aménagements lorsque les divers phénomènes en jeu on conduit à une morphologie relativement stabilisée, comme par exemple dans la Vilaine : les relevés qui avaient été faits en 182 par Beautemps-Beaupré, et ceux faits par la suite, montraient une situation des fonds à peu près stable; en réalité, la stabilité devait n'être que relative, et masquée par certaines variations de caractère plus ou moins cyclique.

Cette faible évolution ne permet pas de recourir, aussi commo. dément que dans le cas de la Seine, à la «méthode historique qui, à ma connaissance, est le meilleur moyen pour «étalonner * un modèle physique et notamment sédimentologique.

Il faut retenir de tout cela que nous disposons maintenant de modèles perfectionnés, qu'il s'agisse de modèles physiques ou qu'il s'agisse de modèles mathématiques; mais, ils ne valent que pour autant que nous introduisons dans ces modèles des données mesurées in situ, conformes à la réalité; il ne faut done pas lésiner sur la collecte de ces données.

En ce qui concerne l'aménagement des estuaires maritimes pour répondre à la demande du trafic, le problème que se posent les autorités portuaires est celui de la nécessité de leur approfondissement, toujours plus important, afin de leur permettre le transit des navires de plus en plus grands.

- La leçon que nous pouvons tirer de cette séance c'est que les «bons vieux principes» de solidarité, de continuité, de la nécessité d'une prédominance du jusant sur le flot, semblent confirmés par quelques dizaines d'années - sinon de siècles d'observations et de réflexion.

- Une autre leçon est la nécessité, lorsqu'on fait des aménagements par des ouvrages, de garder présente à l'esprit la notion d'échelle. Sur un plan, la digue projetée au Verdon, à l'extrémité de la Gironde, apparait comme un «petit» ouvrage dans cet «immense» estuaire; la modification à l'écoulement hydraulique qu'elle peut apporter est relativement faible; elle n'a, en fait, d'effet que dans son voisinage immédiat et n'influence en rien le chenal et le modelé général des fonds.

Le choix de la solution pour aménager un estuaire dépend de l'importance relative de la modification qui doit être apportée à sa configuration naturelle : si la situation naturelle est relativement stable, ou si la modification qui doit lui être apportée est relativement très faible (par exemple approfondissement de quelques mètres d'un chenal de $300 \mathrm{~m}$ de large dans un estuaire de 4 ou $5 \mathrm{~km}$ de large), il est préférable d'agir par dragage; au contraire, la construction d'ouvrages de calibrage est en général la meilleure solution éventuellement combinée avec des dragages - dès lors que leur construction change sensiblement la disposition des lieux et les conditions d'écoulement.

- La dernière leçon de cette séance me paraît être la nouvelle dimension que donnent à l'hydraulique maritime les préoccupations de l'environnement. Nous l'avons vu notamment pour le barrage d'Arzal car c'est probablement là, du fait de la fermeture complète de la Vilaine, que l'aménagement de l'estuaire à comporté le plus grand bouleversement de la situation naturelle. Il est certain que dans des cas semblables - et même si le bouleversement doit être moins important - nous devons de plus en plus nous intéresser, au-delà des problèmes d'écoulement liquide et des transports solides, à l'aspect écologique du problème. Raison de plus pour que vous participiez nombreux à la séance de demain matin et que vous fassiez honneur à l'heureuse initiative de la Société Hydrotechnique de France de réserver une séance entière à l'écologie. Le programme de cette réunion est suffisamment varié pour nous intéresser tous à des titres divers, d'autant plus que la qualité des orateurs garantit celle des exposés.

En remerciant les participants à la présente séance, le Président clôt la discussion.

Après la séance, un film - très applaudi - a été présenté De Bordeatux à la mer, par M,-E. LESPINE. 